\title{
24
}

\section{Dual-view oblique plane microscopy}

Hugh Sparks $^{1}{ }^{1}$ Lucas Dent $^{2}$, Chris Bakal $^{2}$, Axel Behrens ${ }^{3}$, Guillaume Salbreux ${ }^{4}$, Chris Dunsby ${ }^{1,5}$

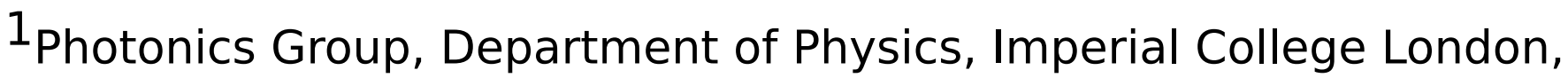
United Kingdom. ${ }^{2}$ Dynamical Cell Systems Team, The Institute of Cancer Research, London, United Kingdom. ${ }^{3}$ Cancer Stem Cell Team, The Institute of Cancer Research, London, United Kingdom. ${ }^{4}$ The Theoretical Physics of Biology Laboratory, The Francis Crick Institute, London, United Kingdom. ${ }^{5}$ Centre for Pathology, Faculty of Medicine, Imperial College London, United Kingdom

\section{Abstract Text}

Light-sheet fluorescence microscopy (LSFM) $)^{1,2}$ provides optically sectioned fluorescence imaging with low photobleaching and photoxicity to the sample ${ }^{3}$. Through rotation of the specimen relative to the LSFM optics it is possible for multiple views of the specimen to be obtained that can be merged in post processing ${ }^{3}$.

The immediate benefits of multi-view imaging are that views can be merged to achieve a more isotropic spatial resolution and that it can be used to overcome artefacts due to sampled induced scattering and absorption 4,5 .

While LSFM traditionally uses separate objective lenses to provide sheet-like laser illumination for fluorescence excitation and to provide orthogonal fluorescence detection, the method of oblique plane microscopy (OPM) enables LSFM using a single microscope objective to illuminate the specimen and collect the resulting fluorescence ${ }^{6}$ and has been developed for near-video-rate 3D fluorescence imaging 7,8 and for high-content imaging of multi-well plates by stage-scanning 9 . 
While OPM has fewer constraints in terms of sample preparation and the ability to easily image large arrays of specimens in multiwell plates, it does not have the benefits of multi-view LSFM. We present a novel, folded OPM configuration called dual-view OPM (dOPM) that enables two separate orthogonal views of the specimen to be achieved. Only a single mechanical actuator is required in order to scan the light sheet and detection plane through the specimen and to switch between the two orthogonal views. This approach enables the benefits of dual-view SPIM e.g. ${ }^{10}$ to be obtained when performing OPM.

We describe the principles of the novel optical design and how the acquired data is reconstructed into the lab frame. For data preprocessing, reconstruction, fusion and deconvolution we present a pipeline based on ImageJ and the multi-view fusion plugin ${ }^{11}$ where volumes are registered based on sub-resolution bead data taken for each view.

To quantify spatial resolution of the dOPM system, volumes of $0.2 \mu \mathrm{m}$ TetraSpeck ${ }^{T M}$ Microspheres embedded in 1\% agarose were recorded and full width half maximum (FWHM) values for line profiles along each axis of the primary microscope Cartesian coordinates are found to determine lateral and axial resolution. While fused bead volumes showed a slight degradation in spatial resolution, deconvolved volumes show a clear improvement in resolution along each axis and a more isotropic 3D point spread function.

To demonstrate that dOPM can be used to improve spatial resolution and overcome sample-induced artefacts in biologically relevant samples, the system was applied to image a fixed multi-cellular spheroid on the order of $100 \mu \mathrm{m}$ in diameter. The spheroid consisted of WM cells with FITC phalloidin labelled actin. The figure shows central ortho-cuts from each view's data together with an overlay showing that the different views provide complementary image information. 
(a)

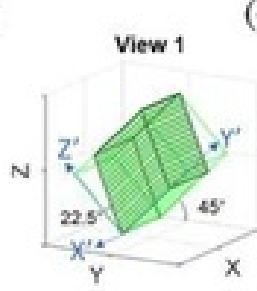

(b)

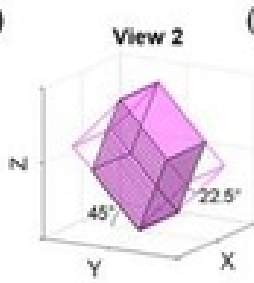

(e)
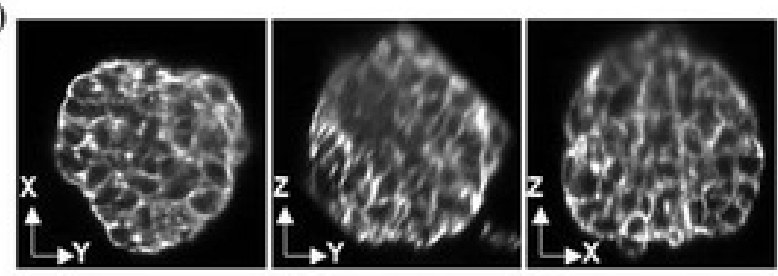

(c)
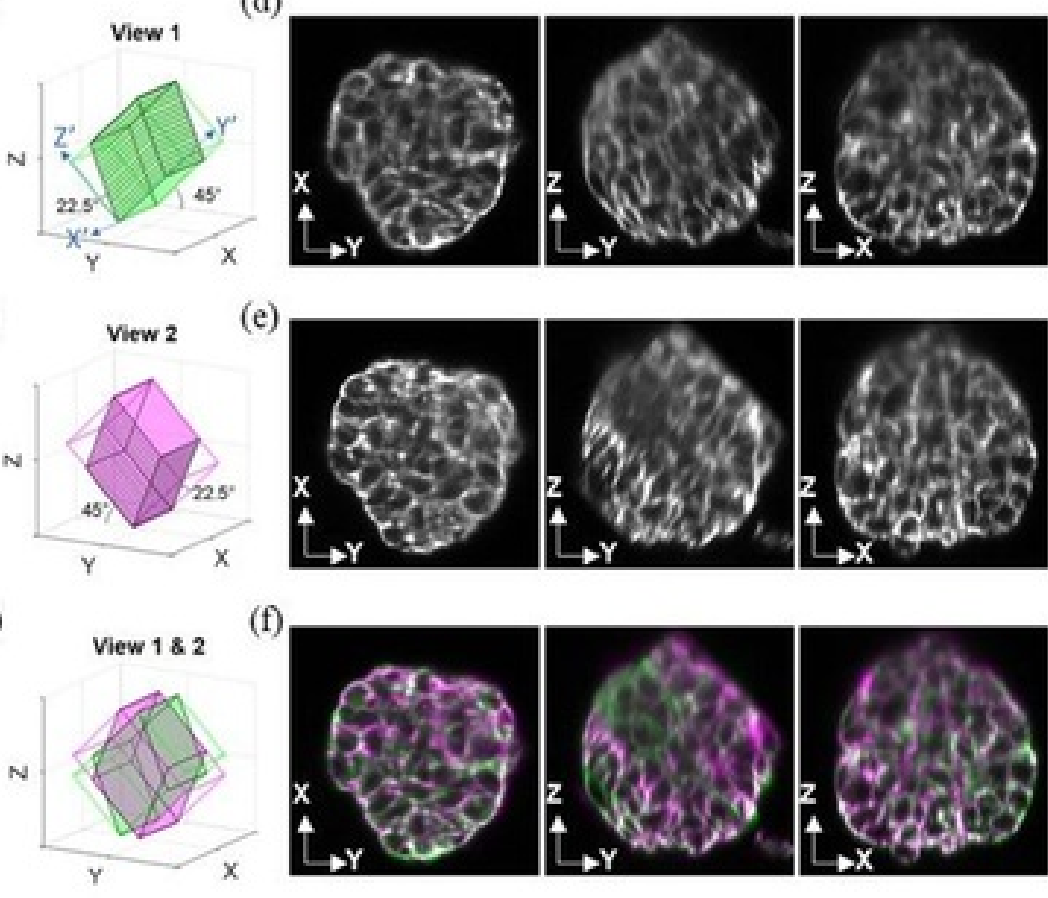

(f)

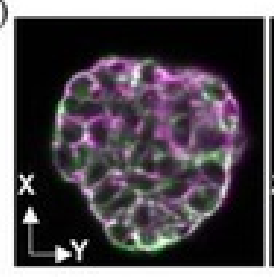

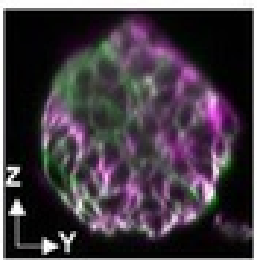

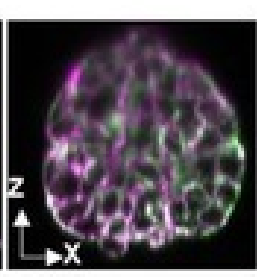

$100 \mu \mathrm{m}$

Figure 1.3D plots (a) and (b) show the outlines of the volumes swept by the two light-sheets in the two views. Plot (c) shows the two views superimposed. Montage (d) and (e) shows orthogonal cuts through dOPM image volumes acquired from a fixed spheroid of WMs cells embedded in Matrigel and where FITC-phalloidin fluorescently labels actin. A $488 \mathrm{~nm}$ laser and a $525 / 50 \mathrm{~nm}$ bandpass emission filter were used for fluorescence excitation and detection respectively. In (d), a montage of three images shows central orthogonal cuts in Y-X planes, Y-Z planes and X-Z planes for View 1. I n (e), equivalent central orthogonal cuts are shown for View 2. In (f), 2-colour overlays are shown for central orthogonal cuts equivalent to (d) and (e) but where the View 1 and View 2 volumes have been co-registered but not fused. Throughout the figure, the Cartesian coordinate systems corresponds to the primary mi croscope where the z-axis is parallel with the optical axis of the primary objective. The scale bar applies to montages in (d)-(f). 